\title{
Animal-derived-antibody generation faces strict reform in accordance with European Union policy on animal use
}

Criticism has plagued data derived from immuno-analysis because of the frequently reported unreliable nature of animal-derived antibodies ${ }^{1,2}$. Polyclonals suffer from cross-reactivity, high background and batch-to-batch variability. The lack of specificity reported in monoclonals is partly attributed to genetic heterogeneity introduced during hybridoma generation and lack of sequence identification. The use of poorly characterized antibodies has a resounding impact in wasted cost, time and resources, and the repercussions on diagnosis and health management are immeasurable.

Advances in recombinant technologies have emerged over the last few decades to engineer antibodies without the need for animal immunizations. Such methods capture the diversity characteristic of the natural immune system by replicating antibody genes from donor B cells or by using precisely designed synthetic gene sequences followed by yeast or phage display for selection of the antigen-specific antibodies. Yet despite calls for change, improved validation strategies and government investment in large development programs ${ }^{1,3,4}$, the dependence on animal-derived antibodies has not really changed. In all scientific sectors except therapeutics, where animal-free technologies are firmly established, scientists appear to accept the shortcomings of antibodies produced using animal immunizations.

However, there is hope on the horizon now that the European Commission's Joint Research Centre has released the much-anticipated Recommendations on Non-Animal-Derived Antibodies from the European Union (EU) Reference Laboratory for alternatives to animal testing $(\text { EURL ECVAM })^{4}$. These recommendations are supported by an in-depth scientific review and opinion on the "scientific validity of replacements for animal-derived antibodies" conducted by its Scientific Advisory Committee, both published as annexes to the recommendation. This publication will influence the future of antibody production in the EU and beyond through EU's global network.

EU policy abides by Directive 2010/63/ EU on the protection of animals used for scientific purposes, which prohibits the use of animals where alternative methods exist $^{5}$. Concerning antibody generation, the requirements of the directive have, until now, been overlooked ${ }^{6}$. However, recent firming up of EU policy ensures that this cannot persist. A major impact on project license applications for animal immunization procedures is anticipated for monoclonal, polyclonal and immunized recombinant antibody production. Applications for animal use will have to be presented with robust justification. Given that antibodies are used in all life science disciplines, that the value of the global antibody market is greater than $\$ 1$ billion annually (therapeutics excluded), and that the number of animals used in antibody generation in Europe alone is estimated at about one million per year, these guidelines are expected to have a broad impact from a commercial and academic perspective.

As scientists move toward using advanced non-animal-derived methods for antibody generation, there will be a significant reduction in animal use. The quality of science and its resulting impact on society will improve because recombinant antibodies show high specificity, have a significantly expanded range of applications and are routinely sequenced, thus allowing fast and reliable identification and reproduction.

Since regulatory authorities will apply the guidance in the Joint Research Centre's report, the use of animal-derived antibodies in regulatory procedures such as diagnostics or safety testing, even when using animal-derived antibodies developed outside of Europe, will be discouraged. As a point of historical relevance, strict EU policy led to a well-established ban on animal testing for cosmetic purposes, which had an extensive impact on the cosmetics industry. This ban also applies to cosmetics imports from any country wishing to trade with Europe. Many countries outside of Europe followed suit by introducing their own restrictions. Similar approaches have seen in vitro methods for toxicity testing replace the use of animals in regulatory procedures, such as a battery of in vitro skin sensitization tests that replace the mouse local lymph node assay ${ }^{7}$.
A similar impact of EU policy is anticipated for the future of antibody development and production. Outside Europe and in response to the EU report and recommendation, a strategy is already being planned by the US National Institutes of Health ${ }^{8}$. The use of animal-derived antibodies in regulatory toxicity procedures is also being addressed at the Organization for Economic Co-operation and Development (OECD) ${ }^{9}$.

A new era is upon us where neither the scientific nor ethical shortcomings of animal-derived antibodies need be tolerated any longer in the interest of a three-Rs principle for antibody production: rapid, reliable and reproducible science. $\mathrm{A}$ poignant and pertinent example of this is the rapid generation of animal-free human monoclonal antibodies to SARS-CoV-2 by biotech companies worldwide, in response to the urgent need for solutions to counteract the spread of the virus. While some groups rely on immunization strategies, others use non-animal approaches, including human B cells from convalescent patients or large pre-existing naive antibody libraries for phage, yeast or ribosome display, permitting the generation of antibodies in as little as four weeks ${ }^{10}$.

Alison C. Gray ${ }^{1 凶}$, Andrew R. M. Bradbury², Achim Knappik ${ }^{3}$, Andreas Plückthun ${ }^{4}$, Carl A. K. Borrebaeck ${ }^{5}$ and Stefan Dübel ${ }^{6}$ ${ }^{1}$ School of Veterinary Medicine and Science, University of Nottingham, Loughborough, UK. ${ }^{2}$ Specifica, Santa Fe, NM, USA. ${ }^{3}$, Bio-Rad, Puchheim, Germany. ${ }^{4}$ Department of Biochemistry, University of Zürich, Zürich, Switzerland. ${ }^{5}$ CREATE Health Cancer Center, Department. of Immunotechnology, Lund University, Lund, Sweden. ${ }^{6}$ Department of Biotechnology, Technische Universität Braunschweig, Braunschweig, Germany.

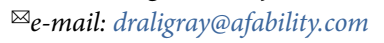

Published online: 27 July 2020 https://doi.org/10.1038/s41592-020-0906-9

\footnotetext{
References

1. Bradbury, A. \& Plückthun, A. Nature 518, 27-29 (2015).

2. Bradbury, A. R. M. et al. MAbs 10, 539-546 (2018).

3. Goodman, S.L. N. Biotechnol. 45, 9-13 (2018).

4. Barroso, J., Halder, M. \& Whelan, M. EURL ECVAM recommendation on non-animal-derived antibodies. EUR 30185
} 
EN (Publications Office of the European Union, 2020); https://doi.org/10.2760/091625

5. European Union. Directive 2010/63/EU of the European Parliament and of the Council of 22 September 2010 on the protection of animals used for scientific purposes (2010) https://eur-lex.europa.eu/eli/dir/2010/63/oj

6. Gray, A. C., Sidhu, S. S., Chandrasekera, P. C., Hendriksen, C. F. M. \& Borrebaeck, C. A. K. Trends Biotechnol. 34 960-969 (2016).

7. OECD. OECD Guidelines for the Testing of Chemicals, Section 4 Test Guidelines 442D and E (OECD Publishing, 2018); https://doi. org/10.1787/20745788

8. Groff, K., Allen, D., Casey, W. \& Clippinger, A. ALTEX 37, 309-311 (2020).
9. Edwards, A. et al. ALTEX 35, 477-488 (2018).

10. The Antibody Society. Will SARS-CoV-2 introduce a new era for subunit vaccines? https://www.antibodysociety.org/coronavirus/ will-sars-cov-2-introduce-a-new-era-for-subunit-vaccines/ (2020).

Competing interests

A.R.M.B.: CSO, founder and shareholder of Specifica Inc., involved in the supply of antibody library and discovery services for the therapeutic market. S.D.: shareholder of companies that employ animal-free antibody generation namely, $\mathrm{mAb}$-factory $\mathrm{GmbH}$, Yumab $\mathrm{GmbH}$ and Abcalis GmbH. A.C.G.: Founder of AFABILITY, a non-profit organization that advises on antibody production methods and scientific procedures involving the use of animals according to Directive 2010/63/EU. A.P.: cofounder and shareholder of companies (MorphoSys AG, Molecular Partners AG) that employ animal-free antibody and binding protein generation. All authors are independent advisors on the EU ECVAM scientific advisory committee (ESAC) for the scientific validity of alternative methods for antibody production. ESAC is composed of external scientists who are appointed on the basis of their scientific expertise; they act independently, in the public interest, and do not represent their company's, the EU's or external interests of any kind. 